\title{
Investigation of Vertical Displacement Thermal Actuators
}

\author{
Naresh Dhaubanjar ${ }^{1}$, Smitha M. N. Rao ${ }^{1}$, Hsu Lun-Chen ${ }^{1}$, Matthew Luquire ${ }^{2}$, \\ Dan Popa ${ }^{1}$, Mu Chiao ${ }^{3}$, Harry Stephanou ${ }^{1}$ and J.-C. Chiao ${ }^{1,2}$ \\ 1. Automation \& Robotics Research Institute, 2. Department of Electrical Engineering, \\ The University of Texas at Arlington, USA \\ 3. Department of Mechanical Engineering, The University of British Columbia, Canada
}

\begin{abstract}
In this paper, we present design, modeling, fabrication, testing techniques and experimental verification for a bidirectional thermal actuator. The actuation principle is based on the asymmetrical thermal expansion of pseudo-bimorph microstructures due to the difference in the electrical resistance of two stacked poly-silicon layers. Bi-directional actuation is achieved depending upon the application of currents on either the top or bottom layers. Various designs were fabricated using the commercial foundry process PolyMUMPS and characterized with a reflective microscope and an optical profiler. Previous demonstrated designs had a limited vertical displacement due to the mechanical limitation imposed by the flexural lengths of the actuator arms. We proposed a new design allowing an increase of the maximum displacement by $85 \%$ with the same input voltage of $7 \mathrm{~V}$. The flexure arm is incorporated in the top silicon layer such that the torsion forces on the flexural arms are minimized. This enables larger deflection of the actuator arm without significant increase in the temperature. Different device configurations have been designed and tested. The temperature distributions on the actuator arms and displacements of the actuators at various conditions were analyzed using finiteelement analysis and verified experimentally. We will discuss the design configuration, testing techniques and practical issues. The potential applications of the out-of-plane actuators include flow sensors, variable capacitors, resistive sensors, optical switches and RF switches.
\end{abstract}

Key words: Thermal actuator, out-of-plane actuator, bi-directional actuation.

\section{INTRODUCTION}

The fields of MEMS (micro-electro-mechanical systems) and optical microsystems are at a fast growing stage. Optical MEMS has become a promising technology in the fields of telecommunication, high-resolution display, and medical imaging. Various actuation mechanisms have been used to actuate the micro optical devices such as electromagnetic, electrostatic, and piezoelectric actuation [1-3]. The thermal actuator can provide larger deflection and higher force [4]. It can be fabricated using cost effective conventional semiconductor processes such as SOI (silicon on insulator), bulk micromachining and surface micromachining.

The actuation principle of thermal actuator is based on the asymmetrical thermal expansion of pseudo-bimorph microstructures due to the difference in the electrical resistance of two stacked poly-silicon layers. Bi-directional actuation is achieved depending upon the application of currents on either the top or bottom layers.

\section{DESIGN}

A typical in-plane thermal actuator consists of a hot arm and a cold arm [5]. The hot arm being thinner than the cold arm, has higher electrical resistance, and therefore provides higher temperature when electrical current is applied. Consequently, the hot arm expands more than the cold arm due to difference in thermal expansion resulting in the actuation mechanism. The out-of-plane thermal actuator is similar to the work done by Yen et al [4]. This design is a modified design of [6] to provide a bi-directional actuation mechanism.

A 3-D view of the vertical thermal actuator is shown in Fig. 1. The actuator consists of two polysilicon layers mechanically connected to each other at one end through via, and anchored to the substrate at the other ends where

Smart Structures, Devices, and Systems III, edited by Said F. Al-Sarawi, Proc. of SPIE Vol. 6414, 641420, (2007) · 0277-786X/07/\$15 - doi: 10.1117/12.695958 
electrical current can be applied. The Arm 1 in Fig. 2 represents the flexure length of the device. The actuation principle of the vertical thermal actuator is similar to that in [4] as the deflection of the actuator tip is due to the difference in thermal expansion of the hot and cold arms. The bi-directional actuation is achieved depending upon the application of electric current on specific electrodes. If the current is applied on the contact pads 1 and 2 in Fig. 1, the deflection is upward because the current only pass through the bottom layer which expands due to the increase in temperature, whereas if electrical current is applied on the contact pads 3 and 4 , the deflection is downward

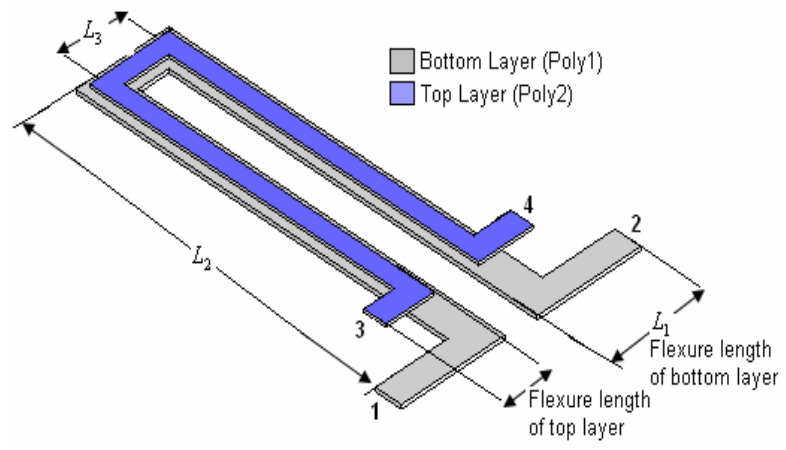

Figure 13 -D view of a bidirectional vertical thermal actuator.

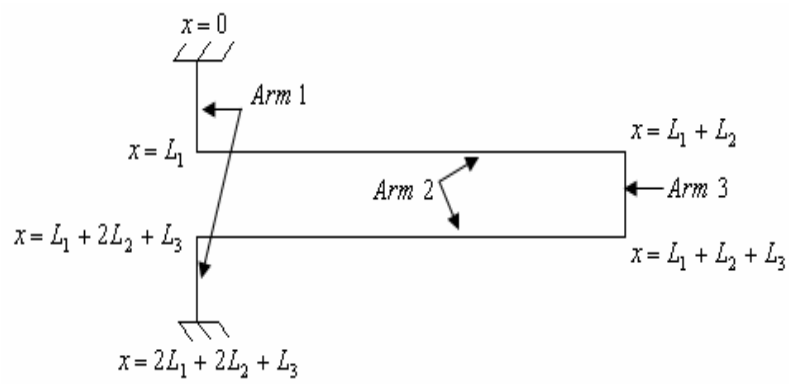

Figure 2 One dimensional representation of the thermal actuator [4].

\section{LUMPED MODELING ANALYSIS}

The lumped modeling analysis is based on the work done by Yen et al. [4, 5]. Assuming the heat loss due to radiation is negligible, the analysis is performed as a one-dimensional heat transfer problem. The temperature distribution along the length of top or bottom layer of the actuator is

$$
T_{i}=T_{s}+\frac{B_{i}}{A_{i}{ }^{2}}+C_{i} e^{A_{i} x}+C_{i}^{\prime} e^{-A_{i} x}
$$

where $i(=1,2$, and 3$)$ represent the number of arm of either the top or bottom layer of one dimensional model as shown in Fig. 2. The parameters are:

$$
\begin{aligned}
& A_{1}=\sqrt{\frac{S_{1}}{k_{p} R_{1} t_{p 2}}+B_{1} \xi}, A_{2}=\sqrt{\frac{S_{2}}{k_{p} R_{2} t_{p 2}}+B_{2} \xi}, \quad A_{3}=\sqrt{\frac{S_{3} w_{2}}{k_{p} R_{3} A_{v}}+B_{3} \xi} \\
& B_{1}=\frac{V_{1}^{2}}{L_{1}^{2} \rho_{0} k_{p}}, \quad B_{2}=\frac{V_{2}^{2}}{L_{2}^{2} \rho_{0} k_{p}}, \quad B_{3}=\frac{V_{3}^{2} A_{v}}{L_{3}^{2} \rho_{0} k_{p} A_{c}} \\
& V_{1}=\frac{V}{2\left(L_{1}+L_{2}\right)+L_{3}} L_{1}, \quad V_{2}=\frac{V}{2\left(L_{1}+L_{2}\right)+L_{3}} L_{2}, \quad V_{3}=\frac{V}{2\left(L_{1}+L_{2}\right)+L_{3}} L_{3}
\end{aligned}
$$

where $S_{i}$ is the shape factor of the arm $i, R_{i}$ is the thermal resistance between the arm $i$ and the substrate, $\xi$ is the linear temperature coefficient, $t_{p 2}$ is the thickness of top layer, and $V$ is the voltage applied on the contact pad.

The temperature distribution along the bottom layer of the thermal actuator can be calculated using Eq. 1-4 and a series of equations given in [4]. The temperature distribution at an input voltage of $5 \mathrm{~V}$ is shown in Fig. 3. Since a lumped model was assumed in the analysis, three separate curves were obtained for each of the three arms of the thermal 
actuator (the blue curves in Fig. 3). A polynomial curve, shown in the red color, shows the actual temperature distribution along the layer. It can be seen from the figure that the temperature distribution is symmetrical about the center. Similarly, the actuator tip deflection can be obtained by using linear thermal expansions of the top and bottom layers as an input to the mechanical analysis. Mechanical analysis similar to [4] can be performed to obtain the total tip deflection of the thermal actuator.

A maximum tip deflection of $6.2 \mu \mathrm{m}$ at an input voltage of $7 \mathrm{~V}$ was reported by Yen et al.[4]. We modified and optimized the flexure design to increase the deflection. We achieved an average tip deflection of $11.5 \mu \mathrm{m}$ at an input voltage of $7 \mathrm{~V}$ which is approximately $85 \%$ increase in total displacement. In this design, the flexure length is $20 \mu \mathrm{m}$ with a length $L_{2}$ of $217 \mu \mathrm{m}$. The higher deflection of the actuator can be achieved without significant increase in the actuator body temperature.

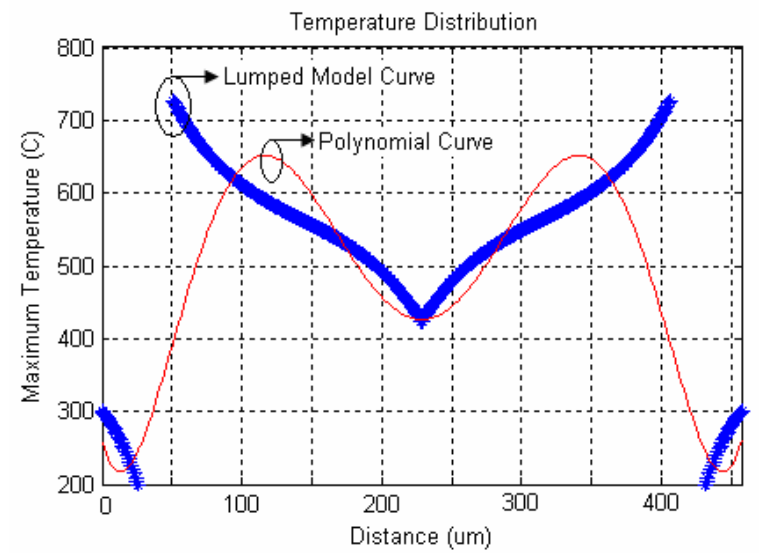

Figure 3 Temperature distributions along the bottom layer of the thermal actuator.

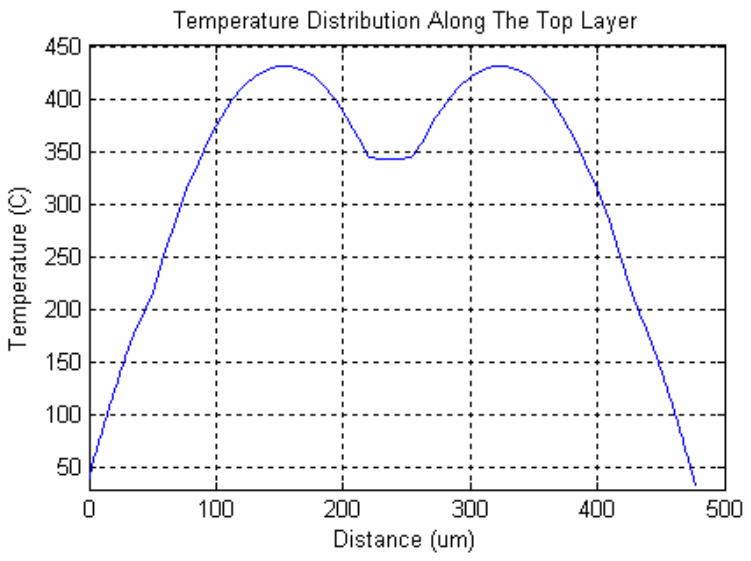

Figure 4 Temperature distributions along the top layer of the thermal actuator.

\section{FINITE ELEMENT ANALYSIS}

Finite element analysis was used to validate the temperature distribution along the top and bottom layers, and actuator deflection. The actuator model was built using SolidWorks and imported to Ansys. A couple field element called "Scalar Tet 98" in Ansys was used for the simulation. Three boundary conditions were applied to the model. Thermal boundary condition was applied by keeping the actuator at an initial temperature of $27^{\circ} \mathrm{C}$. Electrical boundary condition was applied by an input voltage. Mechanical boundary condition was applied by applying zero degree of freedom on the contact pads.

First, the model provided in [4] was simulated to verify our simulation procedure. The simulation result is shown in Fig. 4 at an input voltage of $5 \mathrm{~V}$. The maximum temperature of the top layer obtained from the simulation is $731.56^{\circ} \mathrm{K}$ which is close to the temperature of $695.36^{\circ} \mathrm{K}$ reported by [4]. The electrical parameters used for this simulation are obtained from the MatWeb database [7] as shown in Table 1. The difference in temperatures may be due to the difference in electrical parameters.

Table 1. Material properties of polysilicon.

\begin{tabular}{|ll|}
\hline \multicolumn{1}{|c|}{ Material Properties of Polysilicon } & \multicolumn{1}{c|}{ Value } \\
\hline Young's Modulus & $168 \times 10^{3} \mathrm{MPa}$ \\
Poisson's Ratio & 0.22 \\
Thermal Expansion Coefficient & $3.5 \times 10^{-6} /{ }^{\circ} \mathrm{C}$ \\
Thermal Conductivity & $150 \times 10^{6} \mathrm{pW} / \mu \mathrm{m}{ }^{\circ} \mathrm{C}$ \\
Resistivity & $2.3 \times 10^{-11} \Omega-\mu \mathrm{m}$ \\
\hline
\end{tabular}


Similar procedure is carried out to obtain the temperature distribution for the modified designs at input voltage of $5 \mathrm{~V}$ by varying the flexure length. Fig. 5 shows the simulation result and Fig. 6 shows the temperature distribution along the bottom layer for various configurations of thermal actuators.

Fig. 7 delineates the plots of maximum temperature as a function of input voltage. It can be seen from the plots that there is no significant increase in temperature with decrease in the flexure length of the top layer. Fig. 8 delineates the plots of maximum displacement as a function of input voltage for various flexure lengths. It can be seen from the plot that the actuator with a flexure length of $20 \mu \mathrm{m}$ has a maximum displacement of $10.88 \mu \mathrm{m}$ at an input voltage of $7 \mathrm{~V}$ which is $4.7 \mu \mathrm{m}$ greater than the one with a flexure length of $50 \mu \mathrm{m}$ [4]. Short flexure length minimizes the torsion force on the flexural arms, which in turn maximizes the vertical displacement of the thermal actuator.

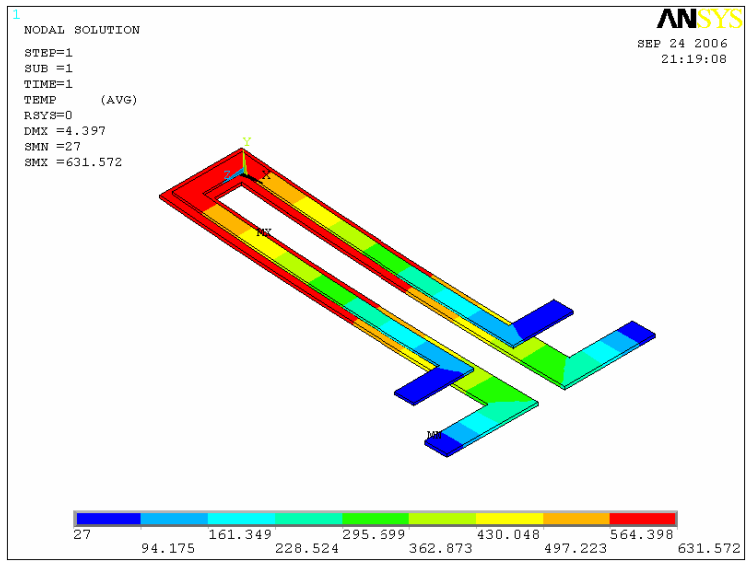

Figure 5 Ansys simulation result.

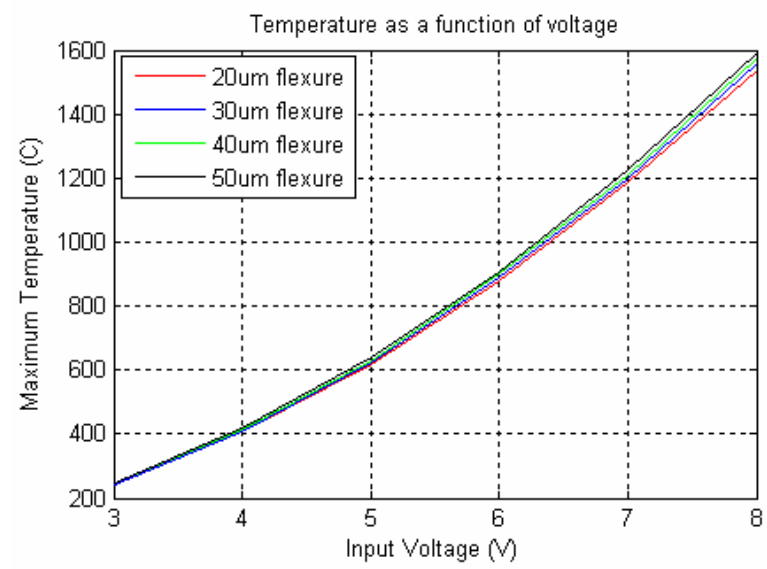

Figure 7 Plots of maximum temperature as a function of input voltage.

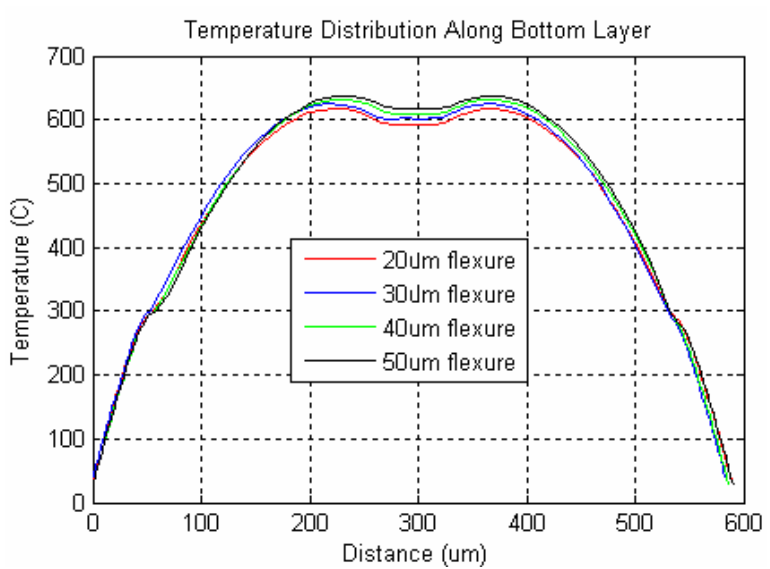

Figure 6 Temperature distributions along the bottom layer of actuators with flexure lengths of $20 \mu \mathrm{m}, 30$ $\mu \mathrm{m}, 40 \mu \mathrm{m}$, and $50 \mu \mathrm{m}$.

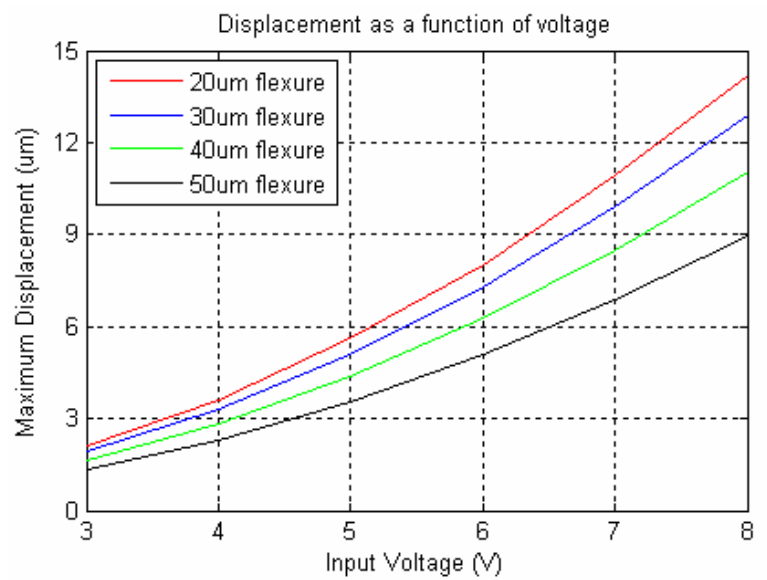

Figure 8 Plots of maximum displacement as a function of input voltage.

\section{EXPERIMENTAL RESULTS}

Various configurations of vertical thermal actuator were fabricated using the PolyMUMPS foundry process. The device dimensions are given in Table 2. The PolyMUMPS chips were released using our in-house HF release and drying process. The scanning electron micrograph (SEM) images of the various configurations are shown in Fig. 9. The 
maximum tip deflection of the actuator was determined experimentally using an optical profiler and a reflective microscope. The experimental setup using the optical profiler, VEECO WYKO NT1100 is shown in Fig. 10. The actuation bias was provided using a current amplifier to the contact pads of actuators by probes. The actuator was dynamically analyzed using the $D M E M S$ option available in the optical profiler.

Table 2. Dimensions of the thermal actuator.

\begin{tabular}{|ll|}
\hline \multicolumn{1}{|c|}{ Device Dimension } & \multicolumn{1}{c|}{ Value } \\
\hline Length of the long arm of the top layer & $180 \mu \mathrm{m}$ \\
Length of poly1-poly2 via & $18 \mu \mathrm{m}$ \\
Flexure length of the top layer & $20,30,40$ and $50 \mu \mathrm{m}$ \\
Width of the top layer & $15 \mu \mathrm{m}$ \\
Length of the long arm of the bottom layer & $217 \mu \mathrm{m}$ \\
Flexure length of the bottom layer & $50 \mu \mathrm{m}$ \\
Width of the bottom layer & $18 \mu \mathrm{m}$ \\
\hline
\end{tabular}

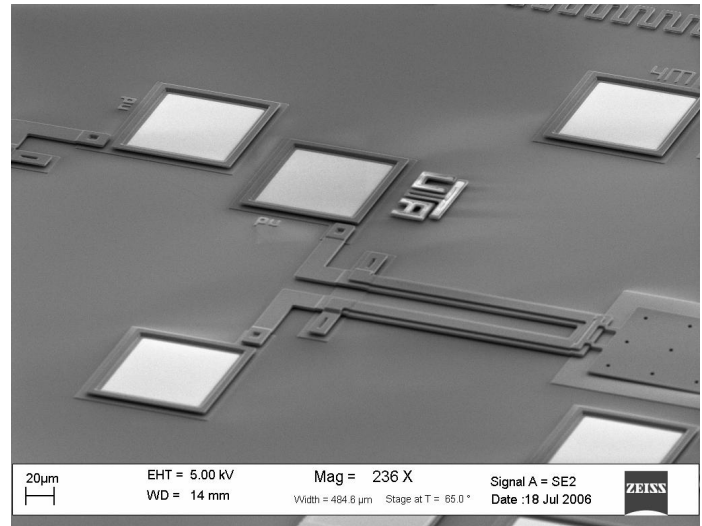

(a)

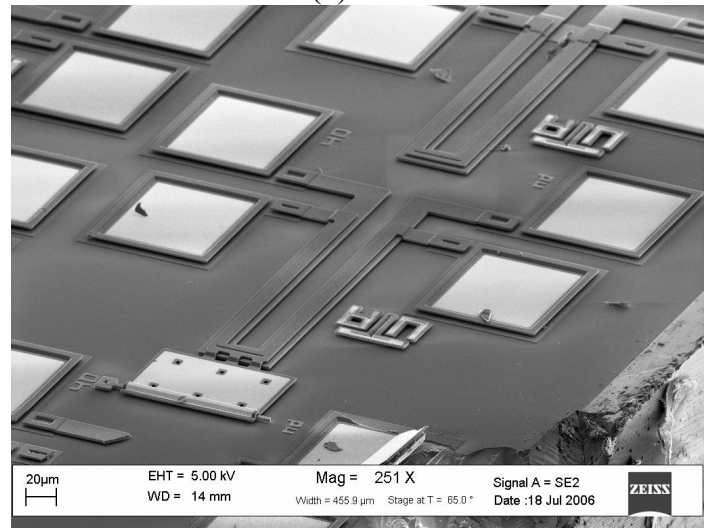

(c)

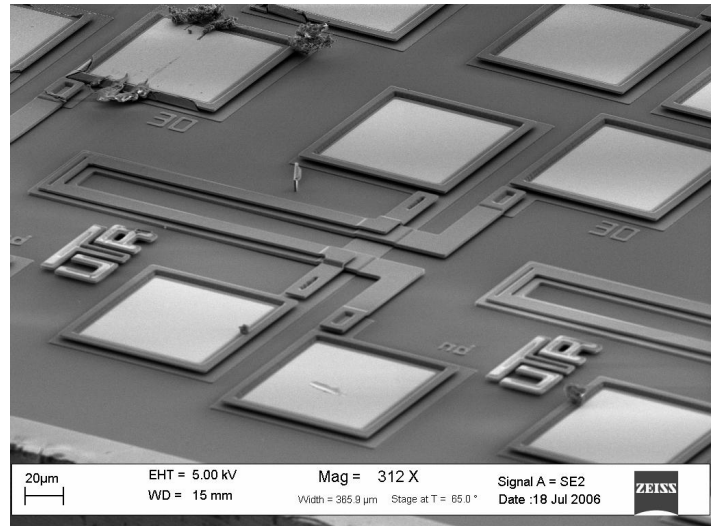

(b)

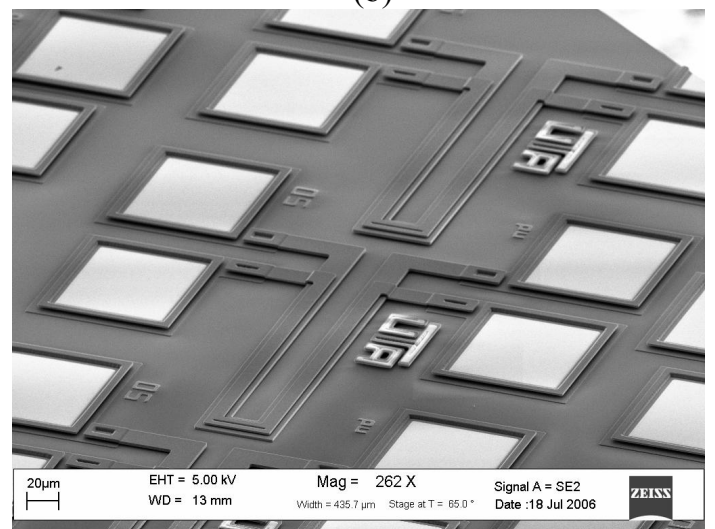

(d)

Figure 9 Scanning electron micrograph images of the vertical thermal actuators with flexure lengths of (a) $20 \mu \mathrm{m}$, (b) $30 \mu \mathrm{m}$, (c) $40 \mu \mathrm{m}$, and (d) $50 \mu \mathrm{m}$.

For the measurement, square waves with amplitudes of $7 \mathrm{~V}$ with a $20 \%$ duty cycle were supplied. The optical reading was taken at every $10^{\circ}$ phase difference. The maximum displacement as a function of phase angle is plotted in Fig. 11. 


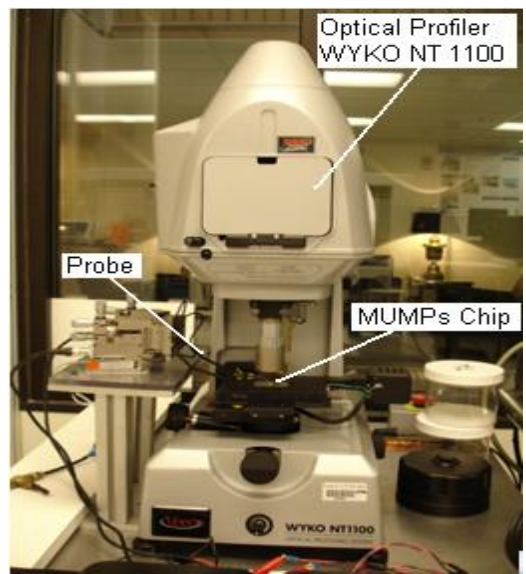

Figure 10 Experimental setup to measure deflection of actuator using an optical profiler.

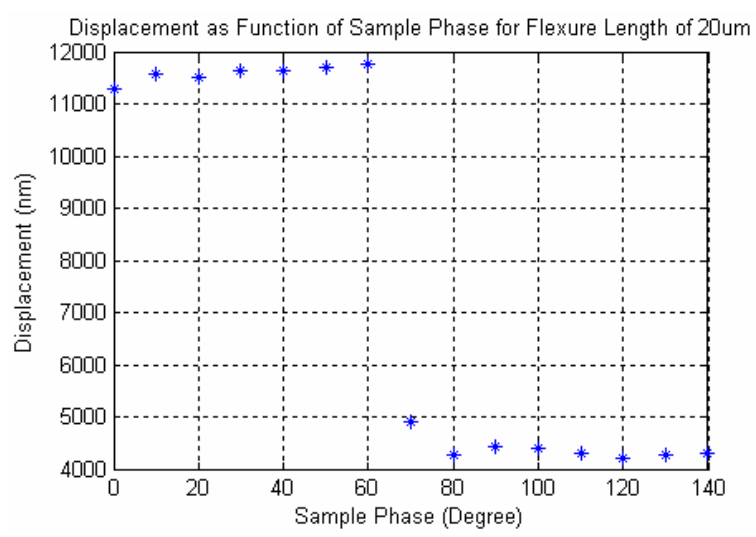

(a)

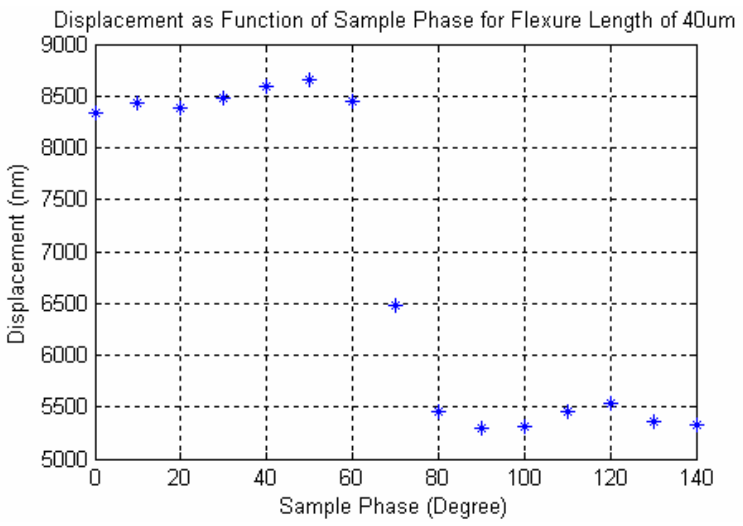

(c)

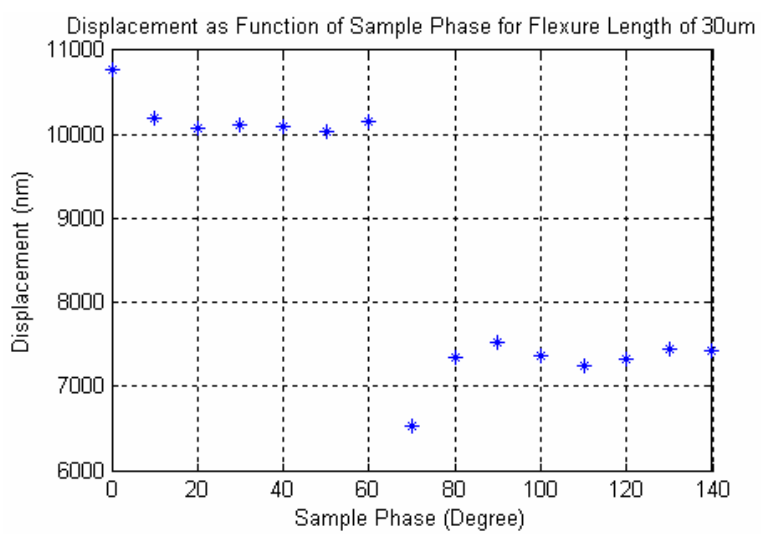

(b)

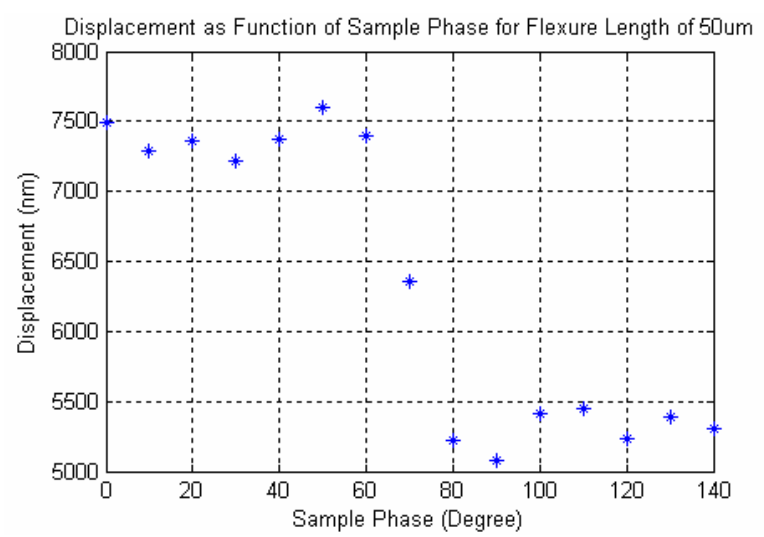

(d)

Figure 11 Maximum displacements as a function of phase angle for actuators with flexure lengths of (a) $20 \mu \mathrm{m}$, (b) 30 $\mu \mathrm{m}$, (c) $40 \mu \mathrm{m}$, and (d) $50 \mu \mathrm{m}$.

It can be seen from the Fig 11 that the thermal actuator with $20-\mu \mathrm{m}$ long flexures has an average displacement of 11.5 $\mu \mathrm{m}$ at an input voltage of $7 \mathrm{~V}$ which is $5.3 \mu \mathrm{m}(85 \%)$ greater than the one with a flexure length of $50 \mu \mathrm{m}$ [4]. Similarly, 
the thermal actuator with 50- $\mu \mathrm{m}$ long flexures has an average displacement of $7.5 \mu \mathrm{m}$ at an input voltage of $7 \mathrm{~V}$. Hence, there is a good agreement between the measured actuator displacement and the finite element analysis.

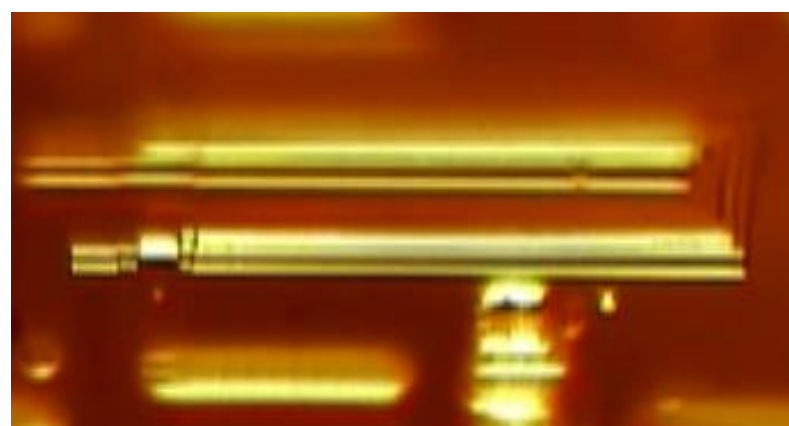

Figure 12 Initial position of the thermal actuator with flexure lengths of $20 \mu \mathrm{m}$ seen from a $45^{\circ}$-mirror.

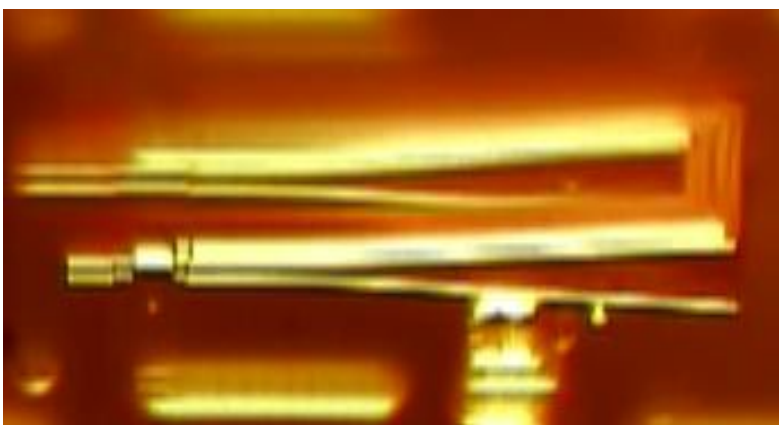

Figure 13 Final position of the thermal actuator with flexure lengths of $20 \mu \mathrm{m}$ seen from a $45^{\circ}$-mirror at an input voltage of $7 \mathrm{~V}$.

The maximum displacement of the thermal actuator can also be obtained using a $45^{\circ}$-mirror under a microscope reflecting the displacement image from the side view. This is to address the concern that the optical profiler uses infrared signals to capture displacement and the heated polysilicon structures may interfere with the optical images. In this method, a CCD camera is focused on the mirror to capture the picture of the actuator deflection. The total deflection is obtained by counting the pixels between the initial and final positions of the actuator. The deflection can also be calculated using simple trigonometric relation, following the method similar to the one in [5]. The initial and final positions of the thermal actuator with flexure length of $20 \mu \mathrm{m}$ are shown in Fig 12 and 13, respectively. The maximum displacement using this measurement method is $11.32 \mu \mathrm{m}$ at an input voltage of $7 \mathrm{~V}$. Both measurement approaches verified the finite element simulation results.

\section{CONCLUSIONS}

In this paper, a bidirectional vertical displacement thermal actuator was presented. The device was fabricated using multilayer polysilicon process, PolyMUMPS. The actuator was characterized using an optical profiler. The actuator was modified to achieve higher displacement without significant increase in the body temperature. The theoretical and experimental results were verified using finite element analysis. The actuator can be utilized to design flow sensors, variable capacitors, resistive sensors, optical switches and RF switches.

\section{ACKNOWLEDGEMENT}

This research is sponsored by the National Science Foundation (NSF) DMI \#0428884. The authors would like to express their gratitude to the NSF support.

\section{REFERENCES}

1. L. K. Lagorece, O. Brand, and M. G. Allen, "Magnetic microactuators based on polymer magnets," Journal of Microelectromechanical System, 8: 2-9, 1999.

2. K. F. Harsh, B. Su, W. Zhang, V. M. Bright, and Y. C. Lee, "The realization and design considerations of a flipchip integrated MEMS tunable capacitor," Sensors and Actuators, A 80:108-118, 2000.

3. Masayuki Kurita, Takanori Yamazaki, Hidekazu Kohira, Masaaki Matsumoto, Ryuuji Tsuchiyama, Junguo Xu, Takeshi Harada, Youichi Inoue, Lizhi Su, and Koji Kato, "An Active-Head Slider With a Piezoelectric Actuator for Controlling Flying Height," IEEE Transactions on Magnetics, Vol. 38, No. 5, 2002.

4. D. Yan, A. Khajepour, and R. Mansour, "Design and modeling of MEMS bidirectional vertical thermal actuator," Journal of Micromechanics and Microengineering, 14:841-850, 2004. 
5. Q-A. Huang and N. K. S. Lee "Analysis and design of polysilicon thermal flexure actuator," Journal of Micromechanics and Microengineering, 9:64-70, 1999.

6. Q-A. Huang and N. K. S. Lee, "Analytical modeling and optimization for a laterally-driven polysilicon thermal actuator," Microsystem Technologies, 5(3):133-137, 1999.

7. http://www.matweb.com 